\title{
BIFENDATE INDIRECTLY INHIBITS ALANINE TRANSAMINASE RELEASE WITHOUT DIRECT INTERACTION IN VITRO AND IN VIVO
}

\author{
HU LI ${ }^{1,2}$, NAN-NAN LIU ${ }^{1}$, JIAN-RUI LI ${ }^{1}$, MEI-XI WANG ${ }^{1}$, JIA-LI TAN ${ }^{1}$, LI-LI ZOU ${ }^{1}$, BIAO DONG ${ }^{1}$, \\ ZONG-GEN PENG ${ }^{1,3 *}$ and JIAN-DONG JIANG ${ }^{1,4 *}$
}

${ }^{1}$ CAMS Key Laboratory of Antiviral Drug Research, Institute of Medicinal Biotechnology, ${ }^{2}$ Key Laboratory of Biotechnology of Antibiotics,

The National Health and Family Planning Commission (NHFPC), Institute of Medicinal Biotechnology, ${ }^{3}$ Beijing Key Laboratory of Antimicrobial Agents, Institute of Medicinal Biotechnology, ${ }^{4}$ State Key Laboratory of Bioactive Substance and Function of Natural Medicines, Institute of Materia Medica,

Chinese Academy of Medical Sciences and Peking Union Medical College, Beijing 100050, China

\begin{abstract}
Bifendate (DDB) is an approved hepatoprotective drug for decreasing serum transaminases in patients with chronic hepatitis in China. However, in some clinical reports, the level of alanine transaminase (ALT) could be rebounded after DDB treatment withdrawal and decreased again once re-treated with DDB, suggesting that DDB might be a reversible inhibitor of ALT or directly inhibit its activity or synthesis. To investigate whether DDB and its metabolites decrease transaminase levels by directly acting on transaminases or how DDB influences the liver transaminases, the dynamics of transaminase activity and protein level were detected after treatment with DDB or with its metabolites in vitro or in vivo. The results showed that DDB reduced the activities and the protein levels of serum ALT and aspartate transaminase in $\mathrm{CCl}_{4}$-induced mice without a significant effect on those in the liver. DDB and its metabolites did not affect the activity elimination rate and the protein degradation rate of ALT, while it significantly reduced the release of transaminases from the damaged primary mouse hepatocytes induced by $\mathrm{CCl}_{4}$. Therefore, DDB does not directly act on transaminases but indirectly decreases transaminase release in vitro and in vivo. Mechanism disclosure of DDB in our study contributes to its security evaluation, clinical practice, and the development of effective drugs derived from DDB.
\end{abstract}

Keywords: Bifendate, liver injury, hepatoprotective, transaminase, mechanism

Hepatotoxicity is a common liver disease caused by extrinsic (e.g., hepatitis viruses, drugs and alcohol) and intrinsic (e.g., ischemia/reperfusion, free fatty acids and toxic bile acids) factors, and it can lead to liver failure and even death $(1,2)$. Up to date, hepatoprotective therapy is still not satisfied, and its treatment is a long-term and arduous task in the clinic $(3,4)$. Bifendate (diphenyl dimethyl bicarboxylate, DDB, Figure 1A), a synthesized derivative of Schizandrin C, is an approved hepatoprotective drug used for the treatment of various chronic hepatitis with elevated alanine transaminase (ALT) in China $(5,6)$. A large number of clinical and preclinical pharmacological studies have proved its effect on reducing serum ALT and aspartate transaminase (AST) in chemi- cal-, drug- and immunological-induced liver injury with high safety $(5,7-10)$. The effect of DDB on reducing ALT is obvious, while the effect on AST has been reported with conflicting results $(5,11$, 12). DDB indirectly inhibits the extracellular release of transaminase from hepatocytes with several unclarified mechanisms, such as preservation of the hepatocyte integrity, an increase of the cellular membrane fluidity, maintenance of the structure and function of mitochondria, and promotion of the protein synthesis (13-15). However, in some clinical reports, transaminases could be rebounded after the withdrawal of DDB and decreased again once re-treated with DDB, suggesting that DDB might be a reversible inhibitor of ALT or directly inhibit its activity or synthesis $(12,16)$. Here, we detected the

\footnotetext{
* Corresponding author: e-mail: (Z.-G.P.) pumcpzg@126.com; (J.-D.J.) jiang.jdong@163.com
} 
dynamics of transaminase activity and protein level after treatment with DDB or with its metabolites in vitro or in vivo to explore how DDB influences the liver transaminases.

\section{EXPERIMENTAL}

\section{Materials}

Bifendate was from Beijing Union Pharmaceutical Company (Beijing, China) with purity over 99\%. Carbon tetrachloride $\left(\mathrm{CCl}_{4}\right)$ was purchased from Tianjin Fuchen Chemical Reagent Factory (Tianjin, China), sodium carboxymethyl cellulose (CMC-Na) was from Sinopharm Chemical Reagent Co. Ltd. (Shanghai, China). Dulbecco's modified Eagle's medium (DMEM) and fetal bovine serum (FBS) were obtained from Gibco (Grand Island, NY, USA). ALT and AST activity assay kits (Nanjing Jiancheng Bioengineering Institute, Nanjing, China), mouse ALT and AST ELISA kits (Cloud-Clone Corp., Wuhan, China), BCA protein assay kit (Thermo Scientific, Waltham, MA, USA), Hanks' balanced salt solution (Beyotime Biotechnology, Jiangsu, China), and type IV collagenase (Sigma, St. Louis, MO, USA) were used in the experiments. Male KM mice (9-10 weeks old, $22.0 \mathrm{~g} \pm 2.0 \mathrm{~g}$ ) with SPF grade were from Beijing HuaFuKang Biological Technology Co. Ltd (Beijing, China).

\section{Animal experiments}

Forty male KM mice were randomly divided into four groups (control group, DDB group, $\mathrm{CCl}_{4^{-}}$ treated group, and $\mathrm{CCl}_{4}+\mathrm{DDB}$ group) with ten mice in each group. The DDB-treated group was intragastrically administrated with $200 \mathrm{mg} / \mathrm{kg}$ DDB twice daily ( $8 \mathrm{~h}$ interval) for 5 consecutive days. After the last administration, $0.1 \% \mathrm{CCl}_{4}$ peanut oil solution was intraperitoneally injected at $10 \mathrm{~mL} / \mathrm{kg}$ body weight. The same amount of solvent $(0.5 \%$ CMC-Na or peanut oil) was treated in the corresponding control groups. Sixteen hours after the injection, the mice were sacrificed for blood and liver tissue collection. The blood samples were centrifuged at $2500 \mathrm{~g}$ for $10 \mathrm{~min}$ and the serum was collected for transaminase protein and activity assay. The liver tissues were stored at $-80^{\circ} \mathrm{C}$ for liver homogenate and subsequent experiments. Animal experiments were conducted following the National Guidelines for Housing and Care of Laboratory Animals and were performed in accordance with the protocol approved by the Institutional Animal Care and Use Committee.

\section{Preparation of serum and liver homogenate con-} taining metabolites of DDB

To prepare the serum and liver homogenate with the highest level of DDB metabolites, ten male KM mice were intragastrically given $300 \mathrm{mg} / \mathrm{kg}$ DDB for twice with $10 \mathrm{~h}$ interval. The serum samples were collected and mixed two hours after the last treatment. Equal amounts of liver homogenate in each mouse after total protein concentration quantification were mixed. Another ten mice were given $0.5 \%$ CMC-Na and treated as above to prepare the control serum or liver homogenate mixture without DDB metabolites.

Isolation of primary mouse hepatocytes and treatment

Primary mouse hepatocytes were harvested from male KM mice according to previously described methods (17-19). Briefly, the mouse was anesthetized
A<smiles>COC(=O)c1cc(OC)c2c(c1-c1c(C(=O)OC)cc(OC)c3c1OCO3)OCO2</smiles>

B

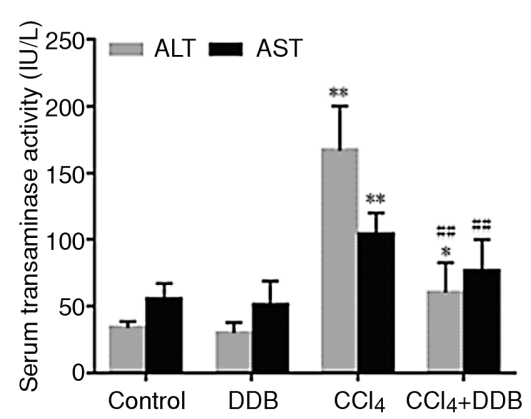

C

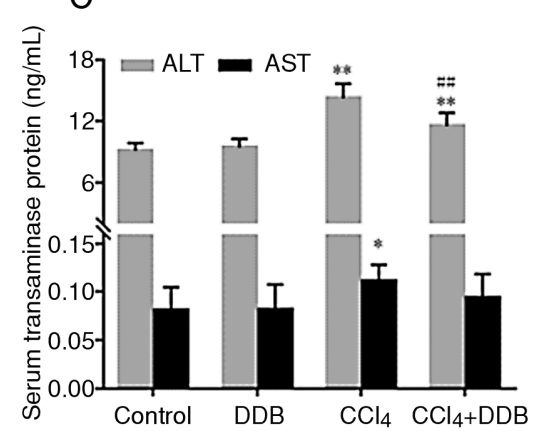

Figure 1. DDB decreases the activity and the protein level of serum transaminases in $\mathrm{CCl}_{4}$-induced acute liver injury in mice $(\mathrm{n}=10)$. $(\mathrm{A})$ The structure of DDB; (B-C) The mice were pretreated intragastrically with $200 \mathrm{mg} / \mathrm{kg}$ DDB and injured by $0.1 \% \mathrm{CCl}_{4}$, serum $\mathrm{ALT}$ and AST activities were detected with transaminase activity assay kit (B), and serum ALT and AST proteins were detected with ELISA assay (C). $* \mathrm{P}<0.05, * * \mathrm{P}<0.01$ vs. control group; \#\# $\mathrm{P}<0.05$ vs. $\mathrm{CCl}_{4}$-treated group. 
A

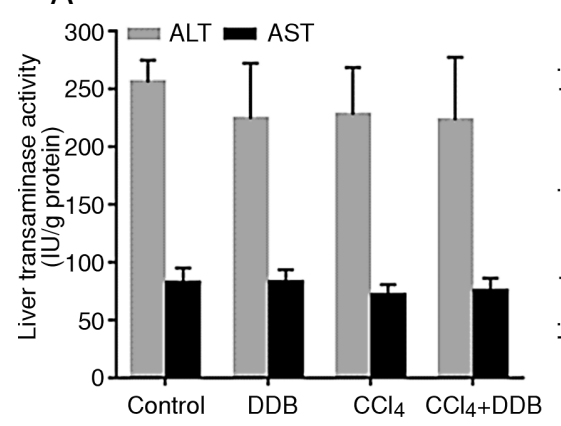

B

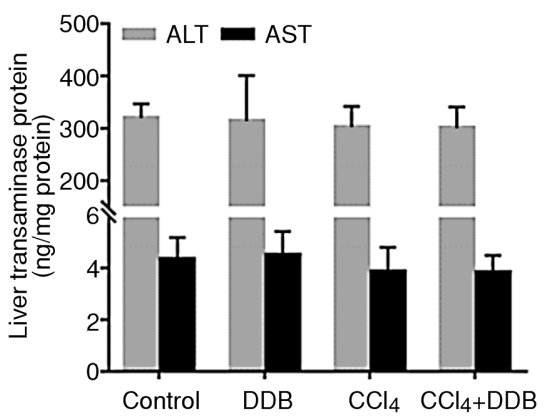

Figure 2. DDB does not alter the activity and the protein level of intrahepatic transaminases in $\mathrm{CCl}_{4}$-induced acute liver injury in mice $(\mathrm{n}=10)$. The mice were pretreated intragastrically with $200 \mathrm{mg} / \mathrm{kg}$ DDB and then injured by $0.1 \% \mathrm{CCl}_{4}$. (A) ALT and AST activities were detected with transaminase activity assay kit after the liver homogenate was adjusted to $0.32 \mathrm{mg} / \mathrm{mL}$ with normal saline; (B) Serum ALT and AST proteins were detected with ELISA method after the liver homogenate was adjusted to $0.01 \mathrm{mg} / \mathrm{mL}$.

and the liver was perfused initially with $10 \mathrm{~mL}$ of $37^{\circ} \mathrm{C}$ pre-warmed sterile $\mathrm{Ca}^{2+}$-free Hank's Buffered Saline Solution (HBSS) containing $25 \mathrm{mM}$ HEPES and $0.5 \mathrm{mM}$ EGTA. Perfusion continued with $40 \mathrm{~mL}$ HBSS containing $1 \mathrm{mg} / \mathrm{mL}$ collagenase (type IV, Sigma). Following perfusion, the liver was incubated in $20 \mathrm{~mL}$ of collagenase solution for $3 \mathrm{~min}$. The detached hepatocytes were filtered with $100 \mu \mathrm{m}$ cell strainer and centrifuged at $100 \mathrm{~g}$ for $2 \mathrm{~min}$. The cell pellets were resuspended in $10 \mathrm{~mL}$ of DMEM containing 10\% FBS and centrifuged again. After cell counting and viability assay using a $0.25 \%$ trypan blue solution, the yielded hepatocytes were plated at the density of $10^{5}$ cells $/ \mathrm{cm}^{2}$ in a 24 -well plate. The culture medium was replaced with fresh DMEM containing $10 \%$ FBS after cell adherence. After growing to $90 \%$, the primary mouse hepatocytes were pretreated with DDB for $12 \mathrm{~h}$. The supernatants were replaced with a saturated $\mathrm{CCl}_{4}$ liver injury culture medium and then collected for the activity measurement of ALT and AST after $4 \mathrm{~h}$ of incubation. To prepare $\mathrm{CCl}_{4}$ saturated Hepatocyte damage culture medium, excessive $\mathrm{CCl}_{4}$ was previously added into DMEM, incubated at $37^{\circ} \mathrm{C}$ in dark for $96 \mathrm{~h}$ and mixed every $24 \mathrm{~h}$.

\section{Detection of transaminase protein and activity}

Transaminase protein levels in serum and liver homogenate were detected with ELISA kit and transaminase activity was detected with a transaminase activity assay kit according to the corresponding instructions. Before the assay for liver homogenate, the total protein level was quantified using a BCA protein assay kit. The transaminase activity in liver homogenate was presented as IU/g total protein, while the transaminase protein level was shown as ng/mg total protein.

\section{Statistical analysis}

The data were presented as the mean \pm standard deviation (SD). Analysis of variance (ANOVA) followed by Student-Newman-Keuls (SNK) post hoc test was performed to compare the different parameters among the groups using SPSS 17.0. The value of statistical significance was set at $\mathrm{p}<0.05$.

\section{RESULTS}

Bifendate (DDB) decreases the activity and the protein level of serum transaminases in $\mathrm{CCl}_{4}$ induced acute liver injury in mice

To confirm the effect of DDB on serum transaminases, the mice with acute hepatotoxicity induced by carbon tetrachloride $\left(\mathrm{CCl}_{4}\right)$ were pretreated intragastrically with $200 \mathrm{mg} / \mathrm{kg}$ DDB administration. The serum ALT and AST activities in the model group (treated with $\mathrm{CCl}_{4}$ ) were significantly increased $(\mathrm{p}<0.01)$, up to $(166.67 \pm 33.53)$ IU/L and (104.09 \pm 15.91$)$ IU/L, respectively (Figure 1B). Certainly, $200 \mathrm{mg} / \mathrm{kg}$ DDB treatment did not affect the serum transaminase activities in normal mice (Figure 1B), indicating that DDB is rather safe. Serum transaminase activities, especially the ALT, were significantly decreased in the DDB-treated mice with acute liver injury induced by $\mathrm{CCl}_{4}$ (Figure $1 \mathrm{~B}, \mathrm{DDB}+\mathrm{CCl}_{4}$ group), which was consistent with previous studies $(5,12,13$, 15). Similarly, DDB did not affect the serum transaminase protein levels in normal mice but alleviated $\mathrm{CCl}_{4}$-induced transaminase proteins release into serum (Figure $1 \mathrm{C}$ ). The results suggested that DDB plays a good protective role in mice against acute liver injury and the decreased 
transaminase activities might be partially due to the decreased serum transaminase protein levels.

DDB does not alter the activity and the expression of intrahepatic transaminases in $\mathbf{C C l}_{4}$-induced acute liver injury in mice

The high level of serum transaminase was mostly derived from the release of transaminase from the damaged liver cells $(20,21)$. Therefore, we further evaluated whether DDB reduced serum transaminase due to its inhibition for the activity or expression of transaminase in the liver. After 200 $\mathrm{mg} / \mathrm{kg}$ DDB pretreatment in the mice with acute hepatotoxicity induced by $\mathrm{CCl}_{4}$, the mouse liver was collected and the activity and expression of intra- hepatic transaminases in the liver homogenate were detected respectively. There were no statistical differences in the transaminase activities (Figure 2A) and transaminase protein levels (Figure 2B) among the four groups. The results indicated that neither $\mathrm{CCl}_{4}$ nor DDB affected the total activities or the protein levels of transaminases in the liver.

DDB and its metabolites do not directly affect the activity elimination and the protein degradation of ALT

To further clarify the mechanism of DDB on the decrease of serum transaminases, we took the ALT as a sensitive indicator to evaluate whether DDB could directly inactivate ALT or accelerate its
A

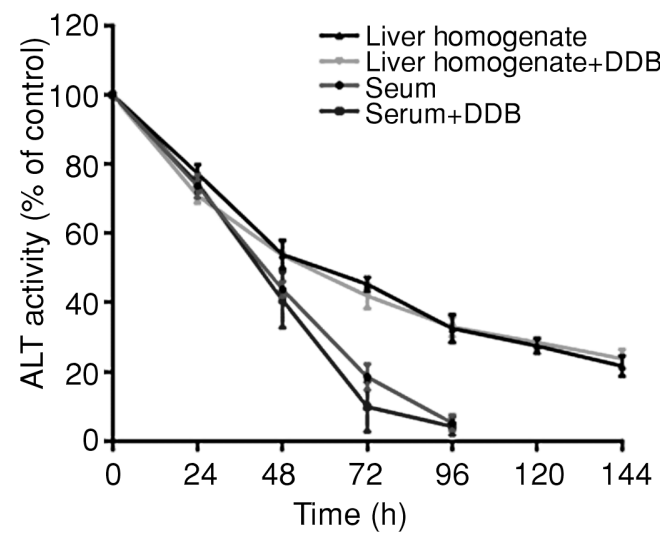

C

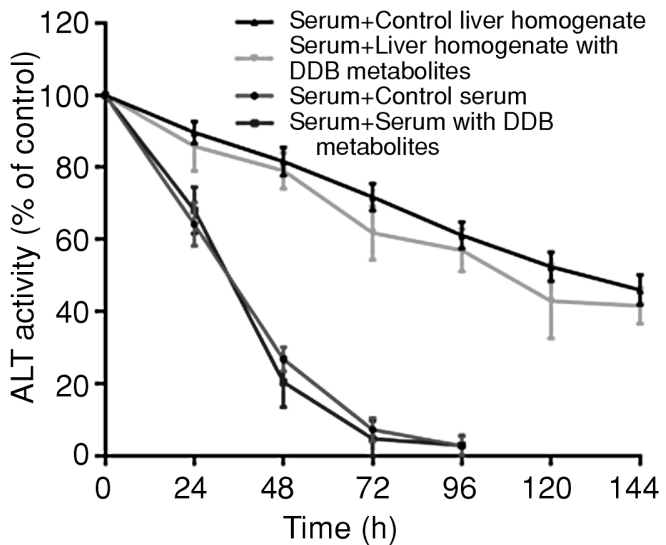

B

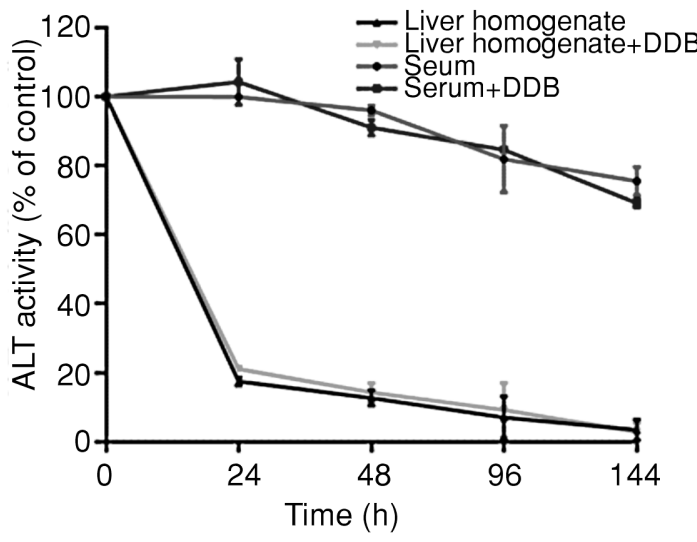

D

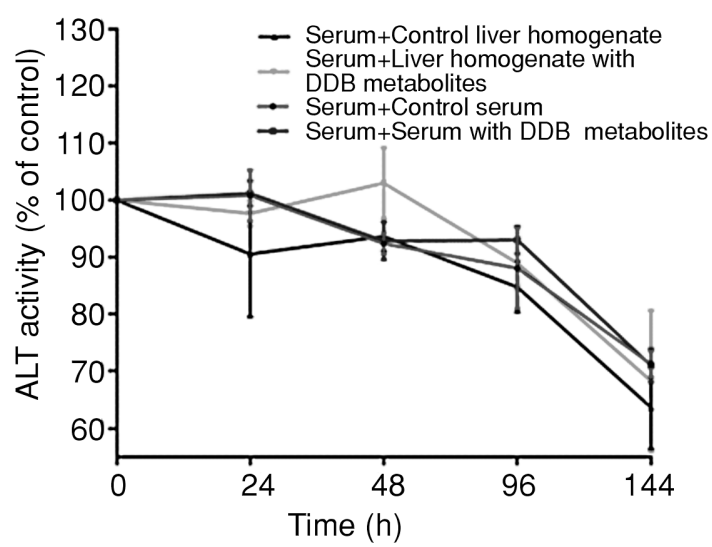

Figure 3. DDB and its metabolites do not directly affect the activity elimination and protein degradation of ALT. Liver homogenate or the serum collected from $\mathrm{CCl}_{4}$-injured mice was incubated with $2 \mu \mathrm{M}$ DDB or solvent for ALT activity (A) and protein (B) detection. The serum collected from $\mathrm{CCl}_{4}$-injured mice was also mixed with liver homogenate $(\mathrm{v} / \mathrm{v}=4: 1)$ or serum $(\mathrm{v} / \mathrm{v}=1: 1)$ derived from DDB treated mice to detect the ALT activity (C) and protein (D) level. For ALT activity measurement, the liver homogenate was adjusted to 0.32 $\mathrm{mg} / \mathrm{mL}$ with normal saline, while it was $0.01 \mathrm{mg} / \mathrm{mL}$ for ALT protein detection. The mixtures were incubated at $37^{\circ} \mathrm{C}$ for the indicated time, and the degradation rate of ALT activity and ALT protein were calculated $(n=3)$. 


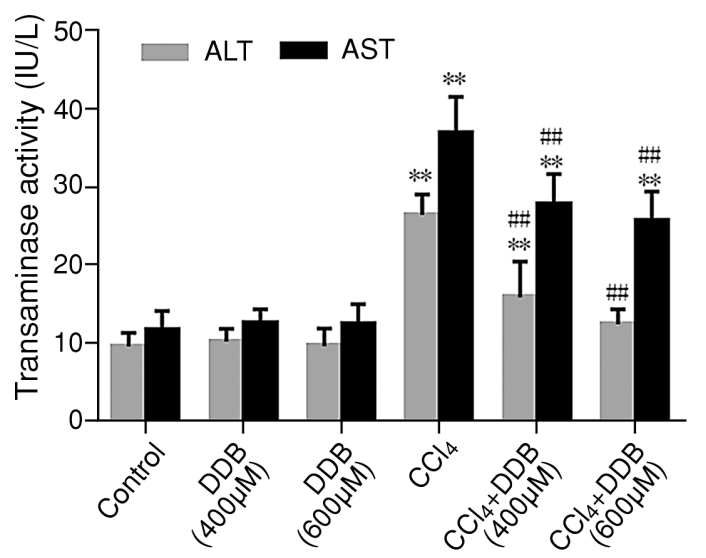

Figure 4. DDB significantly reduces the release of transaminases in $\mathrm{CCl}_{4}$-induced mouse primary hepatocytes in vitro. The primary mouse hepatocytes were isolated from mice and pretreated with DDB for $12 \mathrm{~h}$ and then treated with saturated $\mathrm{CCl}_{4}$ liver injury culture medium for $4 \mathrm{~h}$, the culture medium was collected for the activity measurement of ALT and AST $(\mathrm{n}=3)$. $* \mathrm{P}<0.05$, **P $<0.01$ vs. control group; $\# \mathrm{P}<0.05$, \#\# $\mathrm{P}<0.01$ vs. $\mathrm{CCl}_{4}$-treated group.

activity elimination or protein degradation in vitro. Due to the blood concentration of DDB is generally $0 \sim 4 \mu \mathrm{M}$ after intragastrical administration of DDB in rats at $100-1000 \mathrm{mg} / \mathrm{kg}$ body weight (22) and the half-life of serum ALT was reported for $47 \mathrm{~h}$ (20), we, therefore, chose $2 \mu \mathrm{M}$ DDB for the co-incubation at $37^{\circ} \mathrm{C}$ for consecutive 6 days. After the serum or liver homogenate mixture of the $\mathrm{CCl}_{4}$-treated group was incubated with $2 \mu \mathrm{M}$ DDB or the DMSO solvent, the activity and the protein level of ALT were detected. DDB did not affect the elimination rate of ALT activity (Figure 3A) or the degradation rate of ALT protein (Figure 3B) when compared with the corresponding serum or liver homogenate control, suggesting that DDB itself did not directly act on ALT.

As the effect of DDB is also possibly caused by the DDB metabolites in vivo, we further evaluated the role of DDB metabolites in the activity elimination and protein degradation of ALT. DDB metabolites could be detected in blood one hour after oral administration in mice and reached the peak concentration in the liver and blood at $12 \mathrm{~h}$ (22). Therefore, in this experiment, DDB metabolites in serum and liver homogenates were acquired after intragastrical administration of $300 \mathrm{mg} / \mathrm{kg}$ $\mathrm{DDB}$ in mice. When mixed the serum or liver homogenates with DDB metabolites, no obvious accelerated ALT activity elimination rate (Figure 3C) and ALT protein degradation rate (Figure 3D) were found. Therefore, DDB metabolites may not directly affect ALT activity elimination and protein degradation.
DDB significantly reduces the release of transaminase in $\mathrm{CCl}_{4}$-induced mouse primary hepatocytes

Since DDB and its metabolites did not directly act on transaminase (Figure 2 and 3), the indirect effect on transaminase decrease of DDB in $\mathrm{CCl}_{4}$ induced mouse primary hepatocytes was then verified. The results were shown in Figure 4, although the transaminase activities were low in the normal culture medium, ALT and AST activities were significantly increased in supernatants after $\mathrm{CCl}_{4}$ treatment. DDB significantly reduced the elevated ALT and AST activities induced by $\mathrm{CCl}_{4}$ in a dose-dependent manner (Figure 4), while DDB did not affect the supernatant transaminase activities in the normal mouse primary hepatocytes culture medium. The reduction of ALT by DDB was more obvious, which agreed with the results in our animal experiment and the previous clinical experience $(5,11,12,16)$. The results suggested that DDB might indirectly reduce transaminase release through protecting cellular membrane and mitochondria as reported in previous studies (13-15).

\section{DISCUSSION AND CONCLUSION}

As an approved drug for hepatoprotective therapy in the clinic, DDB could effectively decrease the transaminase levels in various types of liver injury and hepatitis (5, 7-9). However, whether DDB exerts its pharmacologic effects through directly acting on transaminases needs to be clarified. In this study, we confirmed that DDB could reduce the release of transaminases from the damaged hepato- 
cytes, without inactivation of transaminases or promotion of the transaminase activity elimination and affection of the transaminase protein synthesis or degradation by DDB and its metabolites.

DDB effectively reduced the serum ALT and AST levels in our study, especially the serum ALT, which is consistent with previous reports $(5,8,13$, 23). However, some literature also reported that DDB had a poor or ineffective effect on serum AST $(5,11,12)$, which might be related to the severity of the liver injury, the drug dosage, and the treatment duration. DDB can stabilize the hepatocyte membrane, protect mitochondria, and thereby reduce hepatocyte damage or necrosis, leading to an indirect reduction in the release of transaminases (13-15). However, the rebound phenomenon of transaminases after DDB withdrawal in some clinic reports predicted the possibility of DDB as a direct inhibitor of transaminase $(12,16)$. In this regard, Chen detected ALT and AST activity and performed linear regression analysis after gradient dilution of human serum with normal saline or DDB solution (24). He found that the changes in transaminase activity between them were similar, and thus considered DDB might not be a reversible inhibitor of transaminase (24). Regrettably, the real pharmacophore molecules in vivo were most likely DDB metabolites and Chen's work did not include this aspect. Liu et al. showed that ALT activity was not significantly reduced after $2 \mathrm{~h}$ incubation of mice liver homogenates with DDBtreated serum in vitro (9). Moreover, after intravenous injection of serum with high ALT concentration, serum ALT degradation within $8 \mathrm{~h}$ in control and DDB-treated mice were similar (9). Therefore, they speculated that DDB did not directly inhibit the ALT activity or accelerate ALT inactivation. However, serum ALT has a long half-life of about 47 h (20) and it is difficult to observe the significant decrease of ALT activity in a short time. Besides, changes in ALT activity in vivo might also due to the regulatory production or degradation for ALT protein by DDB. Our results showed that DDB decreased the activities and the protein levels of serum transaminases (Figure 1). The damage of a few hepatocytes might cause a sharp increase of serum transaminases (25), and the release of transaminases from the damaged cells had a rather low impact on the intrahepatic total transaminase levels. Therefore, our in vivo results could demonstrate that DDB did not inactivate transaminases or reduce transaminase proteins in mice liver (Figure 2). Moreover, incubation at $37^{\circ} \mathrm{C}$ in vitro excluded the regulatory effect of DDB for cell membranes, mitochondria, and other intracorporal factors. The dynamics of transaminase activity and protein from mouse serum and liver homogenate co-incubated with DDB or its metabolites in vitro therefore further demonstrated that DDB did not accelerate the activity elimination or the protein degradation of ALT (Figure 3).

Hepatocyte damage leads to elevated levels of serum transaminases, among which ALT was mainly present in the cytoplasm and AST was mainly derived from the mitochondria $(20,25,26)$. Based on the reported and our results, the decreased transaminase activity in the cell culture supernatants by $\mathrm{DDB}$ in $\mathrm{CCl}_{4}$-induced primary mouse hepatocytes (Figure 4) is possibly derived from its protective effect on the mitochondria and cellular membrane or other indirect mechanisms (13-15), due to DDB and its metabolites did not directly affect the activity elimination and the protein degradation of ALT (Figure 3). We also speculated the disputed conclusion previously reported about the effect of DDB on AST might be due to the differences in the severity of Hepatocyte damage in vitro and in vivo.

In conclusion, as a hepatoprotective agent, DDB is effective against the release of transaminase from the damaged hepatocytes without direct influence on the activities and protein levels of transaminases by DDB itself or its metabolites. Our study disclosed the mechanism and might contribute to its security evaluation, clinical practice, and the development of new effective hepatoprotective drugs.

\section{Acknowledgments}

This work was supported by the CAMS Innovation Fund for Medical Sciences (2019-I2M1-001), National Natural Science Foundation, China (81621064), and National Mega-Project for "R\&D for Innovative drugs", Ministry of Science and Technology, China (2018ZX09711001-003-010).

\section{Conflict of interest}

The authors declare no conflicts of interest.

\section{REFERENCES}

1. Ding W.X., Yin X.M. (Eds.): Molecules, Systems and Signaling in Liver Injury, Springer, (2017).

2. Tomasz T., Marta D.B., Aneta D.Z., Anna S.: Acta Pol. Pharm. 76, 613 (2019).

3. Domitrović R.,Potočnjak I.:Arch. Toxicol. 90, 39 (2016).

4. Li H., Huang M.H., Jiang, J.D., Peng, Z.G.: World J. Gastroenterol. 24, 5297 (2018). 
5. Zhang Q.,You Y.: Chinese J Drug Eval. 34, 267 (2017)

6. Xie J.X., Zhou J., Zhang C.Z., Yang J.H., Jin H.Q., Chen J.X.: Acta Pharm. Sin. 17, 23 (1982).

7. Gao M., Zhang J., Liu G.T.: Liver Int. 25, 904 (2005).

8. El-Beshbishy H.A.:BMB Reports 38, 300 (2005).

9. Liu G.T., Wei H.L., Song Z.Y.: Acta Pharm. Sin. 17, 101 (1982).

10. Abdel-Hamid N.M.: Afr. J. Biomed. Res. 9,77 (2006).

11. Lee S.H., Cheon G.J., Kim H.S., Kim Y.D., Kim S.G., et al.: Korean J. Gastroenterol. 64, 31 (2014).

12. Huber R., Hockenjos B., Blum H.: Hepatology 39, 1732 (2004).

13. Wang M.R., Jia K.R.: Med. J. Chin. People Liber. Army 15, 436 (1990).

14. Xu Q., Lu J., Wang R.,Wu F., Cao J., et al.: Pharmacol. Res. 35, 273 (1997).
15. Chen L., Chen W.W.: Acta Pharm. Sin. 12, 881 (1988)

16. Tang L.B., He S.M.: J. Practical Hepatol. 185 (2006).

17. Hoffman J.S., Benditt E.P.: J. Biol. Chem. 57, 10518 (1982)

18. Li W.C., Ralphs K.L., Tosh D.: Methods Mol. Biol. 633, 185 (2010).

19. Huang M., Kim H.G., Zhong X., Dong C., Zhang B., et al.: Hepatology 71, 76 (2019).

20. Knapen M.F., Peters W.H., Mulder T.P., Steegers E.A.: Lancet 355, 1463 (2000).

21. Liu Z., Zhang Y., Graham S., Wang X., Cai D., et al.: J. Hepatol. S0168 (2000).

22. Wang X.L., Yi M.G., Liu Z.M., Song Z.Y.: Acta Pharm. Sin. 18, 892 (1983).

23. Lee M.H., Kim Y.M., Kim S.G.: Int. J. Clin. Pharmacol. Ther. 50, 778 (2012).

24. Chen S.H.: Flight Surgeon. 12 (1996).

25. Kew M.C.: Lancet 355, 591 (2000).

26. Kim H.G., Huang M., Xin Y., Zhang Y., Zhang X., et al.: J. Hepatol. 71, 960 (2019).

(C) 2020 by Polish Pharmaceutical Society. This is an access article under the CC BY NC license (http://creativecommons.org/licenses/by-nc/4.0/). 